\title{
Motor imagery effectiveness for mirror reversed movements
}

\author{
Ursula Debarnot • Gaetano Valenza • \\ Stéphane Champely • Enzo Pasquale Scilingo • \\ Danilo De Rossi • Aymeric Guillot
}

Published online: 19 November 2010

(C) The Psychonomic Society, Inc. 2010

\begin{abstract}
Physical practice is known to enhance motor adaptation skills, which refer to the individual ability to compensate for environmental changes. So far, it is still unknown whether a similar effect can be observed following motor imagery (MI). Thirty-nine participants were tested during a joystick tracking task under both normal and mirror conditions (i.e., the inductive direction of the joystick was reversed), before and after a physical practice or MI training phase. Eye movements and electromyographic activity were recorded during MI. Motor performance was also evaluated after a $6 \mathrm{~h}$ interval during daytime. As compared to the control group, the results revealed that both MI and physical practice improved motor performance in the mirror condition, during the posttraining test. Furthermore, the time to complete the task was further reduced after 6 hours, both in the normal and mirror conditions. These results demonstrate the effectiveness of MI for learning mirror-reversed movements, and for the consolidation process that follows motor adaptation.
\end{abstract}

Keywords Motor adaptation $\cdot$ Mental practice $\cdot$ Mirror tracking task $\cdot$ Memory consolidation $\cdot$ Mental chronometry Eye movements

U. Debarnot $(\bowtie) \cdot$ G. Valenza $\cdot$ E. P. Scilingo $\cdot$ D. De Rossi Interdepartmental Research Centre E. Piaggio, School of Engineering, University of Pisa,

Via Diotisalvi 2,

56126, Pisa, Italy

e-mail: ursula.debarnot@gmail.com

U. Debarnot $\cdot$ S. Champely $\cdot$ A. Guillot

Centre de Recherche et d'Innovation sur le sport, EA 647,

Laboratoire de la Performance Motrice, Mentale et du Matériel,

Université Claude Bernard Lyon 1, Université de Lyon,

Lyon, France

\section{Introduction}

Motor skill acquisition is the process by which movements are performed effortlessly through repeated practice and interactions with the environment (Willingham, 1998). Skill acquisition can be characterized by at least two distinct phases: a fast, within-session phase of performance improvement, and a more delayed, latent, time-dependent improvement phase occurring between sessions (Doyon \& Ungerleider, 2002; Hikosaka, Nakamura, Sakai \& Nakahara, 2002). The delayed phase has been conceptualized as reflecting the memory consolidation process, which is the transformation of a given experience into a robust and enduring form (Stickgold \& Walker, 2007). Numerous studies have shown that the motor learning process is composed of motor sequence learning and motor adaptation (MA) (Aizenstein, Stenger, Cochran, Clark, Johnson, Nebes \& Carter, 2004; Doyon \& Benali, 2005; Ghilardi, Eidelberg, Silvestri, \& Ghez, 2003). Motor sequence learning is assessed by incremental acquisition of movements with repetition, while MA refers to the individual ability to compensate for environmental changes. Doyon, Penhune, and Ungerleider (2003) have proposed an integrated view of the plasticity that these two motor memory traces can undergo across learning stages. Accordingly, motor sequence and MA skills might recruit similar cerebral structures during the early learning phase, but would then activate either the cortico-striatal or corticocerebellar anatomical systems following the motor consolidation process. This latter process has been shown to be either stabilized or enhanced during motor sequential (Goedert \& Willingham, 2002; Walker, Brakefield, Hobson, \& Stickgold, 2003) and MA (Richardson, Overduin, Valero-Cabre, Padoa-Schioppa, Pascual-Leone, Bizzi \& Press, 2006) skills, six hours after the initial acquisition 
(Brashers-Krug, Shadmehr, \& Bizzi, 1996; Press, Casement, Pascual-Leone, \& Robertson, 2005).

In the wealth of the motor learning literature, motor imagery (MI) is a valuable complement to physical practice (PP) to enhance cognitive and motor performance (Guillot \& Collet, 2008). MI is the process of mentally rehearsing a motor act without overt body movement (Jeannerod, 1995). In more recent years, MI has also been considered a possible therapeutic tool in rehabilitation of motor disorders (Sharma, Pomeroy, \& Baron, 2006; de Vries \& Mulder, 2007). Interestingly, a large amount of research has provided evidence that MI and PP of the same movement share similar cerebral substrates, although the neural networks are not totally overlapping (Decety \& Jeannerod, 1996; Gerardin, Sirigu, Lehéricy, Poline, Gaymard, Marsault et al., 2000; Solodkin, Hlustik, Chen, \& Small, 2004; Lotze \& Halsband, 2006), hence supporting the principle of functional equivalence (Jeannerod, 1994; Holmes \& Collins, 2001). Consistent with findings from the motor sequence learning literature, Guillot, Collet, Nguyen, Malouin, Richards and Doyon (2008) further demonstrated that compared to skilled imagers, poor imagers not only need to recruit the cortico-striatal system, but compensate with the cortico-cerebellar system during MI of sequential movements. To date, however, the evaluation of MI effectiveness for the consolidation process that follows MA is far less established.

Mirror reversed movements can be considered a MA task (Agostino, Sanes, \& Hallett, 1996; Laforce \& Doyon, 2002), but few experimental studies investigated whether MI might contribute to enhance motor learning of a movement in a reversed configuration. Though not specifically addressing this issue, Koslow (1987) nicely demonstrated that mental practice resulted in better performance in a mirror-drawing test. Based on these preliminary findings, one may hypothesize that MI of a mirror MA task would improve the ability to perform a new pattern of movement. This could be of particular interest when MI is used to acquire patterns of movement including a corrected configuration of actions, or when MI is performed to learn a new pattern of movement, when the physical execution of the movement is no more possible, e.g., following stroke or severe motor impairments. The present study was thus designed to investigate whether performance gains might occur during MA following imagery practice, as it is well established for PP. Based on the functional equivalence between MI and motor performance (Jeannerod, 1994), we hypothesized that these two types of practice would be equally effective in enhancing hand-tracking movements during both a normal MA task, and its mirror condition. To evaluate MI accuracy and control adherence of the participants to the instructions, imagery times, eye movements and muscle activity were recorded (Heremans et al.,
2009; Guillot, Louis, \& Collet, 2010; Personnier, Ballay \& Papaxanthis, 2010). In particular, it has been proposed that eye movements could assist in the process of mental image generation, and therefore reflect the spatiotemporal evolution of the underlying mental processes (Heremans et al., 2009). We therefore compared two experimental situations (one where the participants were free to move their eyes during MI, and another one where they kept the eyes fixed), in order to see whether free eye movements were used to anticipate the direction of the cursor during MI, and whether preventing eyes movements might hinder the MI process.

\section{Materials and methods}

\section{Participants}

A total of 39 healthy volunteers aged between 20 and 37 years (mean age: $27.35 \pm 3.5$ years; 16 women) participated in this study. All were right-handed, as assessed by the Edinburgh Handedness Inventory (Oldfield, 1971), and none had any prior history of drug or alcohol abuse or neurological or psychiatric disorders. They were instructed to be drug, alcohol, and caffeine free for $24 \mathrm{~h}$ prior, and throughout the experiment. Game players spending more than $1 \mathrm{~h}$ to play video games per week were excluded to avoid any expertise effects on pursuing tracking tasks. The procedure of the experiment and the tasks were explained, while no information was provided about the objectives of the study, or the variables of interest. This study was approved by the Research Ethics Committee of the University of Pisa, and all participants signed an informed consent form.

\section{Design and apparatus}

\section{Pursuit tracking task}

A joystick tracking task, where spatiotemporal and dynamic control of the action are required, was chosen as changes in behavioral performance are usually observed following both physical and mental practice (Papadelis, KourtidouPapadeli, Bamidis, \& Albani, 2007; Stephan, Fink, Passingham, Silbersweig, Ceballos-Baumann, Frith et al., 1995). Participants were comfortably seated $60 \mathrm{~cm}$ in front of a $17^{\prime}$ computer monitor (with $1680 \times 1050$ pixel resolution). They controlled the $x$ - and $y$-positions of the yellow round cursor on a computer display screen using a total degree-of-freedom joystick. The joystick was secured to the table in front of the subjects, placed at their body midline. The participants were instructed to track a moving visual target with the joystick-controlled cursor within a 
spatial delimited pathway (length $=48 \mathrm{~cm}$, width $=1 \mathrm{~cm}$ ) as fast and as accurately as possible, while making as few errors as possible (Fig. 1). At the beginning of each trial, the cursor was automatically repositioned in the same location. Two different experimental conditions were randomized: In the normal MA condition, the movements of the joystick (up, down, left, right) were congruent with the displacement of the cursor. In the mirror condition, the inductive direction of the cursor was reversed, i.e. pushing the joystick left resulted in a displacement of the cursor on the right, and vice versa. In a pre-experimental session with an independent group of participants, we checked that movement times were not too rapid or too long, but generally ranged between $7 \mathrm{~s}$ and $15 \mathrm{~s}$ in each experimental condition, though the subjects were asked to perform the task as fast as possible. This time window was preselected to determine the speed of the cursor, as rapid and long movements were frequently found to be respectively overand underestimated during MI, whereas movements lasting between $7 \mathrm{~s}$ and $15 \mathrm{~s}$ were more frequently imagined in real-time (e.g., Guillot \& Collet, 2005; Slifkin, 2008).

In each trial, movement time was recorded, while movement accuracy was determined by the number of contacts with the boundaries of the pathway. Each contact of the cursor was recorded and the cursor was automatically repositioned (half distance of the pathway limits).

\section{MI ability}

All participants were asked to fill out subjective measures of alertness and fatigue using the Stanford Sleepiness Scale (SSS, Hoddes, Dement, \& Zarcone, 1972) during the experimental day. This is a subjective evaluation of the individual level of sleepiness, which consists of seven descriptively and anchored points. The statements reflects

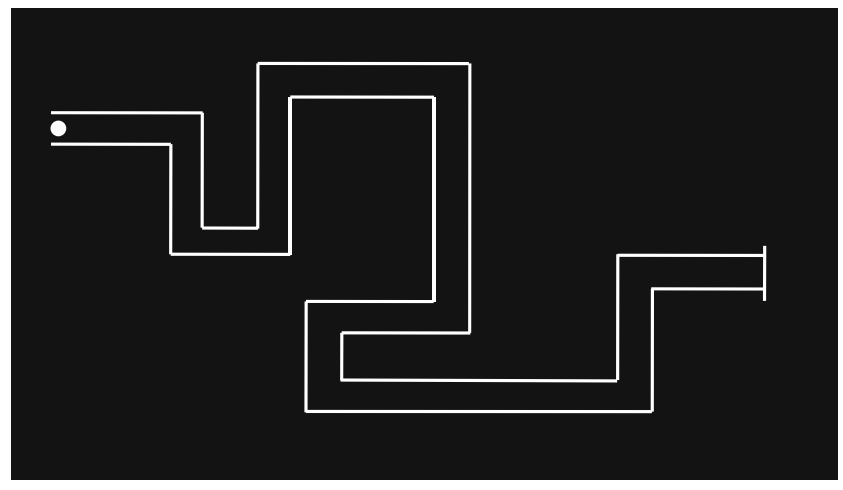

Fig. 1 Pursuing tracking task. The round was controlled with the joystick. The color of the solid lines delimiting the pathway was different in the 'normal' (green) and 'mirror' (red) conditions, to provide a distinct visual feedback to the participant, regarding the task to be physically or mentally performed. A solid line represented the arrival point of the pathway the severity of the sleepiness from "Feeling active and vital; alert and wide awake" to "Almost in reverie; sleep onset soon; lost struggle to remain awake". Despite the retrospective nature of this questionnaire, it has been shown that polysomnographically measuring sleep latency was correlated with the subjective feeling of sleepiness, as measured by the SSS (Bootzin, Manber, Loewy, Kuo \& Franzen, 2004). Two imagery questionnaires were used to evaluate both visual and kinesthetic imagery abilities, as these two forms of imagery were used to mentally rehearse the motor task during the experimental design. The revised version of the Movement Imagery Questionnaire (MIQ-R, Hall \& Martin, 1997) was administered. It is an eight-item self-report questionnaire, in which participants rate the vividness of their mental representation using two sevenpoint scales. The first series of items measures the individual ability to form visual images, while the second rates the ability to perceive the sensations usually elicited by the movement during kinesthetic imagery $(1=$ very hard to see/feel, and $7=$ very easy to see/feel). The participants also completed the revised version of the Vividness of Movement Imagery Questionnaire (VMIQ-2, Roberts, Callow, Hardy, Markland, \& Bringer, 2008) to determine on a five-point scale the vividness of their imagined movements, including the difference between their capacity to use internal and external visual imagery.

\section{Pre-training session}

The experiment was scheduled at 9:00am $( \pm 1$ h). To familiarize the participants with the two experimental conditions, they were given five trials per condition in a randomized mode. Practically, when the lines delimiting the pathway were green, the participants were requested to perform the normal task condition, whereas red lines indicated them to perform the mirror task condition. Following this introductory session, the pre-training session consisted of 30 randomized trials per condition. Two blocks of 15 trials were separated by a period of $60 \mathrm{~s}$ so that the pre-training session lasted approximately $5 \mathrm{~min}$.

\section{Training period}

The participants were assigned to one of four groups that differed in the type of practice to be performed. To ensure that the evolution of the performance would not depend upon individual differences, we checked for similar MI ability in all groups by comparing MIQ-R and VMIQ-2 scores. The PP group $(n=10)$ physically performed four blocks of 30 randomized trials (15 trials in each of the two tracking conditions). The EyesMI group $(n=9)$ was required to imagine the motor task in the two tracking conditions during four blocks of 30 randomized trials. 
During imagery, participants were free to make eye movements at their own convenience. In contrast, participants assigned to the NoeyesMI group $(n=10)$ were instructed to keep the eyes open but to fixate the center of the screen during the same four blocks of 30 imagery trials. These two MI groups rehearsed the MA task as accurately as possible while making as few errors as possible in a quiet room and without any environmental constraints. A MI script was read to ensure that the participants followed the correct instructions throughout the imagery sessions (Appendix). Accordingly, they were requested to combine both visual and kinesthetic imagery, using a first-person imagery perspective. Individual debriefings were scheduled to investigate adherence to the intervention, during which the participants were asked to describe the content of their imagery, to report if they encountered difficulty in forming the mental images, and to explain whether they followed the instructions of the experimental design. During MI, the participants' hand remained motionless at the starting location, as controlled by electromyographic recordings (see below). At the end of the imagery trial, the participants were instructed to press a key on a keyboard with their non-dominant hand to stop the timer, thus allowing the recording of the imagined duration. Finally, the control group $(n=10)$ was subjected to a period of neutral activities (solitaire card games) during equivalent time, without performing PP or MI at all. This neutral activity did not require the same cognitive abilities as those involved in forming mental images. The four blocks were separated by a period of $60 \mathrm{~s}$, so that the training session lasted approximately $10 \mathrm{~min}$.

\section{Post-training tests}

Two different post-training sessions were scheduled to investigate the impact of the training period and the daytime consolidation effects on motor performance. The first post-training session (post-test) was performed immediately after practice and was similar to the pre-test. A second identical post-training session (re-test) was scheduled $6 \mathrm{~h}$ after the first post-test (Korman, Doyon, Doljansky, Carrier, Dagan, \& Karni, 2007). In each case, two blocks of 30 randomized trials were physically performed.

Physiological and behavioral recordings

To control eye movements and to check that arm muscles were inactive during imagined movements, electrooculographic (EOG) and electromyographic (EMG) signals were continuously monitored during MI. Both signals were acquired by means of a Biopac MP150 (Biopac System, CA, USA) with a sample frequency of $1 \mathrm{kHz}$. The EOG signal was recorded on the right eye with two pairs of red- hot $\mathrm{Ag}-\mathrm{AgCl}$ surface electrodes. After skin preparation, one pair of electrodes was adhered nasally and temporally to the eye, and another one above and below the eye. Both horizontal and vertical eye movements were therefore detected. The obtained signal was filtered with a bandpass filter ranging from $0.05 \mathrm{~Hz}$ (low cut-off frequency) to $30 \mathrm{~Hz}$ (high cut-off frequency). Simultaneously, EMG signals were continuously recorded by means of three Ag$\mathrm{AgCl}$ surface electrodes with a diameter of $44 \mathrm{~mm}$, which were placed over the bellies of the extensor and the flexor carpi radialis muscles, following standard skin preparation techniques. The obtained signal was filtered by means of a band-pass filter from $30 \mathrm{~Hz}$ (low cut-off frequency). The EMG was shown into a band between $30 \mathrm{~Hz}$ and $250 \mathrm{~Hz}$. EMG activity and eye movements were controlled online, i. e., when the experimenter observed any activity (except free eye movements in the EyesMI group), the participants were immediately instructed to relax their arm and/or focus on the screen, and the trial was discarded from further analysis.

\section{Data analysis}

The dependent variables were the movement accuracy (number of contacts with the limits of the pathway) and movement times. This latter corresponds to the time needed to complete the pathway, from the onset of the tracking movement to the crossing finish line. Imagery times were also recorded to check whether participants were able to comply with the imagery guidelines. This technique has been shown to be a reliable method to evaluate individual imagery ability (Guillot \& Collet, 2005; Malouin, Richards, Jackson, Lafleur, Durand, \& Doyon, 2007). As no significant gender difference was found when comparing the number of correct sequences, as well as average times, there was no need to consider gender as an independent variable. Regarding the statistical analyses, dependent variables were first log-transformed because of the skewness of their distributions, and the presence of several outliers. Three-way analyses of variance (ANOVAs) with repeated measures were then performed to study the effects of the between-subjects factor (Groups) and the two withinsubjects factors (Sessions and Conditions). Polynomial contrasts were used to break the Session effects into its linear and quadratic components. An a priori set of specific contrasts was employed for describing the group differences. Normality of the residuals was graphically checked using a confidence envelope of the normal quantile plots generated by parametric bootstrap (Atkinson, 1985, computations done using the Rcmdr package of Fox, 2005).

Finally, we used non-parametric statistics (KruskalWallis one-way ANOVA) to compare the results of the SSS and MI test scores, due to their discrete nature. 


\section{Results}

\section{Questionnaires}

There was no group difference when comparing the SSS scores during both the immediate post-training session $(\mathrm{H}=$ $1.99, p>.05)$ and the re-test $(\mathrm{H}=1.85, p>.05)$. On the seven-point scale $(1=$ being most alert), the mean (SD) values were $1.87(.64)$ in the control group during the first post-training session (post-test), and $1.5(.50)$ during the retest. Respective mean scores were $1.42(.53)$ and $1.42(.53)$ in the PP group, $1.6(.74)$ and $1.75(.70)$ in the EyesMI group, and $1.87(.80)$ and $1.25(.46)$ in the NoeyesMI group. Mean MIQ-R scores were 48.7 (4.14) in the control group, 46.2 (3) in the PP group, 43 (3.50) in the EyesMI group, and 45.6 (3.30) in the NoeyesMI control group. As expected, there was no difference between the groups $(\mathrm{H}=$ $2.38, p>.05$ ), but visual imagery scores were higher than kinesthetic imagery scores in all groups $(p<.001)$. Similarly, no difference was found when comparing the average VMIQ-2 scores in all groups $(\mathrm{H}=.91, p>.05)$ with mean scores being 92 (7.80) in the control group, 85.8 (4.80) in the PP group, 82.5 (8.30) in the EyesMI group, and 88 (3.10) in NoeyesMI group. As expected, the participants reported the lowest scores (i.e., greater imagery vividness) during external visual imagery, and the highest scores during kinesthetic imagery, respective mean scores being 26.8 (4.06), 29.44 (4.68), and 30.83 (4.70) during external visual, internal visual and kinesthetic imagery. Altogether, the lack of inter-group difference in the two MI questionnaires guaranteed homogeneity regarding the individual ability to form mental motor images.

\section{Behavioral data}

Comparing movement accuracy among the four groups during the pre-test session did not reveal any difference in the ability to perform the task $(F(3,35)=.8, p=.5)$. Moreover, there was no difference with regards to the time taken to perform the task $(F(3,35)=.68, p=.57)$. We further graphically checked that all participants reached an asymptotic level of performance during the training session to ensure that performance was stabilized before moving to the post-test session.

\section{Movement accuracy}

Mean (SD) numbers of contacts with the limits of the pathway, during both the normal pathway and mirror condition, are summarized in Table 1. The three-way repeated measures ANOVA of the (log) movement accuracy did not show any interaction $(F(6,175)=.7, p=.65)$. Considering the simple effects, only the test of the Sessions factor produced a significant result $(F(2,175)=25.90, p<.001)$. The quadratic component of the effect was clearly significant $(F(1,175)=$ $7.40, p=.007)$ : the mean of pre-training movement accuracy $(1.77 \pm 1.31)$ was higher and the two post-mean values were similar $(1.24 \pm .94$ vs. $1.13 \pm .74)$. A normal quantile plot of the residuals showed no deviation from the normality assumption.

\section{Movement times}

Mean (SD) times taken to perform the MA task are summarized in Table 2. The repeated measures analysis of variance of the movement times demonstrated that the Session by Group was the only statistically significant interaction $(F(6,175)=7.40, p<.001)$ and that the Conditions simple effect was separately significant $(F(1$, $175)=26.10, p<.001)$. The quadratic component of the polynomial contrast of the Sessions factor was not significant leading to a more interpretative linear evolution of the mean movement times. Moreover, a set of specific contrasts was used to understand the Group effects: (1) = mean of the control group vs. mean of the three other groups, (2) = mean of the PP group vs. mean of the two remaining groups and (3) = mean of the EyesMI vs. the NoeyesMI group. Considering the linear component of the Session by Group interaction, the first contrast was significant $(F(1,175)=42, p<.001)$ but the two other contrasts were not. It means that the evolution of the movement times over the three sessions were approximately linear in the four groups, that in each group the profiles of the two conditions were parallel, and that the slopes of

Table 1 Mean (standard deviations) number of errors in the normal and mirror conditions

\begin{tabular}{|c|c|c|c|c|c|c|}
\hline & \multicolumn{3}{|c|}{ Normal condition } & \multicolumn{3}{|c|}{ Mirror condition } \\
\hline & Pre-training & Post-test 1 & Re-test & Pre-training & Post-test 1 & Re-test \\
\hline PP group & $1.67(1.43)$ & $1.14(.80)$ & $.81(.68)$ & $1.54(1.34)$ & $.92(.66)$ & $.75(.44)$ \\
\hline Control group & $1.89(1.72)$ & $1.25(1.36)$ & $1.36(1.16)$ & $1.77(1.65)$ & $1.07(1.21)^{*}$ & $1.19(.67)$ \\
\hline EyesMI group & $2.35(1.25)$ & $1.40(0.80)^{* *}$ & $1.61(.74)$ & $2.22(1.18)$ & $1.77(.86)$ & $1.28(.24)$ \\
\hline NoEyesMI group & $1.41(.89)$ & $1.28(.94)$ & $1.02(.70)$ & $1.44(.89)$ & $1.17(.76)$ & $1.05(.79)$ \\
\hline
\end{tabular}


Table 2 Mean (standard deviations) movement times in the normal and mirror conditions

\begin{tabular}{|c|c|c|c|c|c|c|}
\hline & \multicolumn{3}{|c|}{ Normal condition } & \multicolumn{3}{|c|}{ Mirror condition } \\
\hline & Pre-training & Post-test 1 & Re-test & Pre-training & Post-test 1 & Re-test \\
\hline PP group & $13.5(.77)$ & $12.21(.58)^{* *}$ & $11(.50)^{*}$ & $15.1(1.30)$ & $12.93(.81)^{*}$ & $12.11(.52)^{*}$ \\
\hline Control group & $12.82(.36)$ & $12.46(.34)^{*}$ & $12.81(.36)$ & $13.23(.55)$ & $13.02(.49)$ & $13(.41)$ \\
\hline EyesMI group & $12.64(.65)$ & $11.74(.44)^{*}$ & $11.07(.42)^{*}$ & $13.72(.75)$ & $12.36(.49)^{* *}$ & $11.45(.40)^{*}$ \\
\hline NoEyesMI group & $12.49(.48)$ & $11.46(.27)^{* *}$ & $10.52(.31)^{*}$ & $13.03(.58)$ & $11.54(.43)^{* * *}$ & $10.83(.38)^{*}$ \\
\hline
\end{tabular}

the control group was different from those of the three other groups (Fig. 2). A normal quantile plot with envelope of the residuals showed a very good symmetry in this dataset, and a reasonable fit of the normal distribution (with the exception of three small outliers among 234 values).

\section{MI training times}

During MI training, the EyesMI and NoeyesMI participants took on average $7 \mathrm{~s}(.70)$ and $10 \mathrm{~s}(.50)$ to mentally imagine the MA task in the normal pathway condition, $11.1 \mathrm{~s}(.74)$ and $10.5 \mathrm{~s}(.82)$ in the mirror condition. No significant group difference was observed both in the normal and mirror conditions $(t=.99, p>.05$ and $t=.56, p>.05$, respectively). As compared to the actual pre-test durations, the data revealed that MI times were shortened in the eyesMI and NoeyesMI groups both in the normal $(t=2.52$, $p<.05$, and $t=5.83, p<.001)$ and mirror conditions $(t=$ $2.64, p<.05$, and $t=5.32, p<.001)$.

Assessment of imagery use

\section{Muscular and ocular activities}

The data provided evidence that during imagined movements, all muscles normally involved in the overt movement remained totally inactive, hence demonstrating that the participants were able to rehearse the movement without any overt motor output. Similarly, the data confirm that there was not any eye movement in the NoeyesMI group.
Fig. 2 Mean movement times $(\log )$ evolution during normal and mirror conditions. $P P$ physical practice; $M I$ motor imagery
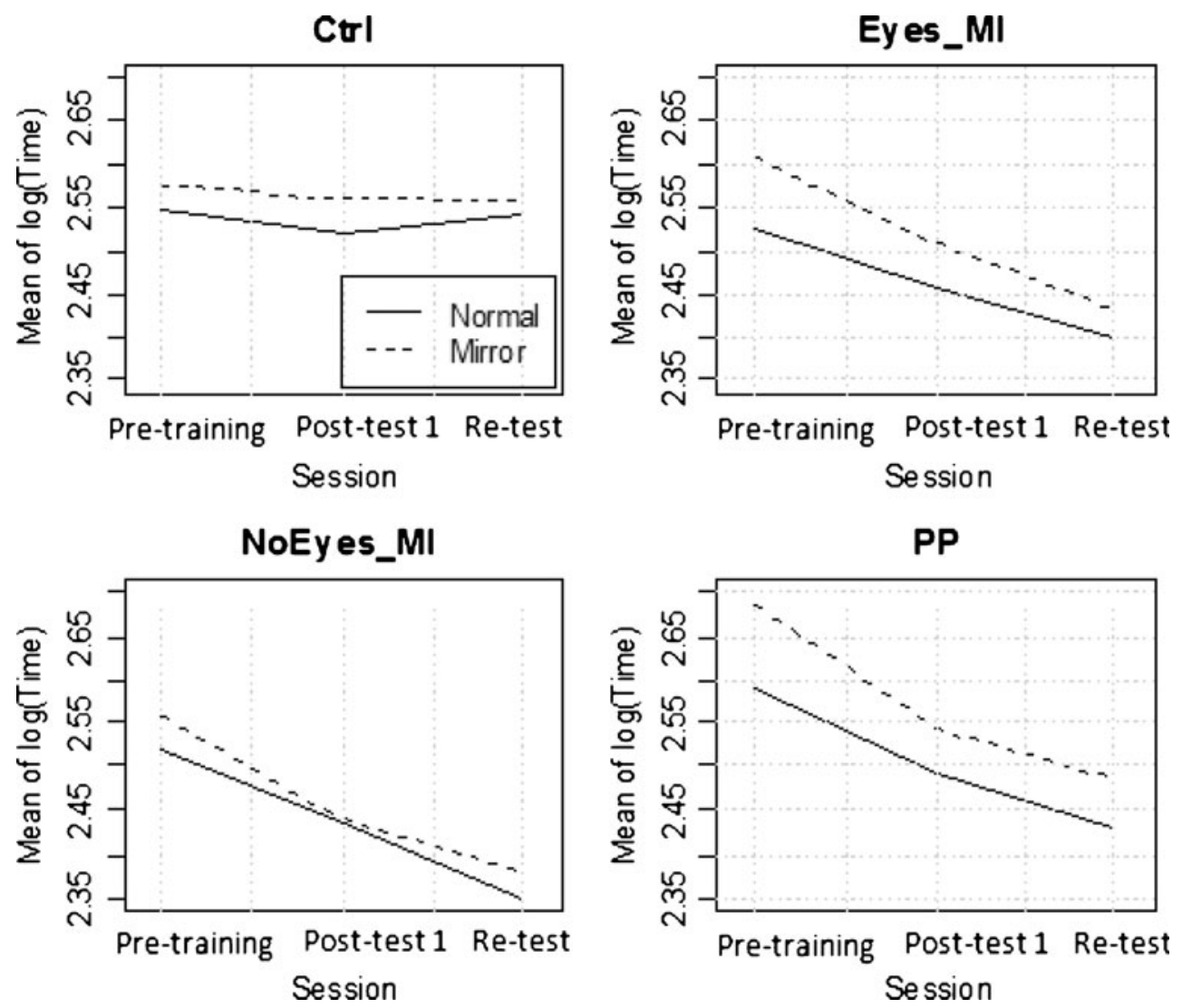


\section{MI debriefings}

Firstly, no group difference was found when comparing the self-reported individual ratings evaluating the vividness of the mental motor images. Mean (SD) scores were $4.4(.20)$ in the eyesMI group and $4.2(.20)$ in the NoeyesMI group. Secondly, during the debriefing sessions following MI, all participants reported that they used the imagery types outlined in the scripts. They adequately combined internal visual and kinesthetic imagery without switching to external visual imagery. The participants reported that they primarily focused their attention on each turn of the pathway (to rehearse changes of the joystick movement), rather than on the straight line. In addition, they reported that this focus was more intense in the mirror condition. Finally, none reported changing the imagery script to suit individual needs, and all participants rehearsed the task as requested. Indeed, they were able to report each movement with an explicit knowledge of what they performed during PP.

\section{Discussion}

The aim of the present experiment was to investigate whether MI practice may contribute to improve motor performance of a mirror MA task. Based on the wellestablished effectiveness of PP (Overduin, Richardson, Bizzi, \& Press, 2008; Seidler, 2004), we hypothesized that MI would similarly contribute to enhance MA performance, which might further be improved following the consolidation process. A main effect of the task was found in all groups, hence suggesting evidence that the mirror condition was significantly more difficult than the normal condition. The results overall showed that both MI and PP training elicited significant improvement in the normal and mirror conditions during the post-training test. Interestingly, the data also revealed that the PP and MI participants further reduced the time to complete the path after $6 \mathrm{~h}$ of consolidation, both in the normal and mirror conditions.

As expected, PP and MI groups improved performance in the normal pathway condition during the posttraining session. Accordingly, the participants subjected to PP and MI training took less time to perform the pursuing tracking task after practice. These results are consistent with those of the motor learning literature, which have extensively demonstrated the effectiveness of MI in improving the spatiotemporal and dynamic control of motor performance (Gentili, Papaxanthis, \& Pozzo, 2006; Papadelis et al., 2007; Yágüez, Nagel, Hoffman, Canavan, Wist, \& Hömberg, 1998; Debarnot, Creveaux, Collet, Doyon, \& Guillot, 2009; Debarnot, Creveaux, Collet, Gemignani, Massarelli, Doyon, \& Guillot, 2009).
In line with the data by Seidler $(2004,2006)$, the present data provide also empirical evidence that PP enhanced motor performance during the post-training test in the mirror MA condition. Interestingly, the same pattern of results was seen following MI practice, hence reinforcing the principle of equivalence between $\mathrm{MI}$ and motor performance (Holmes \& Collins, 2001). These data also support the work by Helene and Xavier (2006), who showed that mental training contributed to improve mirror-reading skill performance as quickly as PP. As we did not provide any visual feedback to the participants during MI, the adaptation procedures usually engaged during the actual performance of a mirror MA task might have been engaged by the maintenance of the mental image within working memory (Farah, 1984), hence contributing to motor skill acquisition. In other words, MI practice improved mirror MA skills through some cognitive manipulation (transformation) of the mental image of the movement to be performed in the normal condition, in order to overcome the constraints of the environment, i.e., by rehearsing a reversed movement of the joystick to keep the correct direction.

A major and innovative finding of this study is that MI practice also resulted in better performance in the mirror condition during the re-test (after $6 \mathrm{~h}$ of daytime), i.e., the participants further reduced the time needed to complete the task. The effectiveness of the consolidation process has been extensively documented in the literature to explain performance gains in both sequential and MA learning (e. g., Bock, Schneider, \& Bloomberg, 2001; Krakauer, Ghilardi, \& Ghez, 1999). Especially, Doyon, Korman, Morin, Dostie, Hadj Tahar, Benali et al., (2009) have demonstrated that the expression of consolidation gains in sequential motor tasks would benefit from sleep, while the simple passage of time might be sufficient in MA tasks. In line with this finding, our results show a benefit from further behavioral consolidation effect in the normal adaptation task for both PP and MI groups after daytime. Interestingly, MI was found to be as effective as PP in improving motor performance in the normal and mirror conditions. While mental practice has rarely been found to outperform PP, Wohldmann, Healy, and Bourne (2007) recently reported that these two types of practice could be equally effective to learn and maintain a new motor skill across a 3-month delay. In a second study, the same authors looked at the effect of a physical interference which was scheduled 1 and 2 weeks following the first learning phase (Wohldmann et al., 2008). The authors concluded that PP would strengthen an effector-dependent representation of the task, while MI should rather strengthen an effectorindependent representation. This could be of particular interest when MI is used to learn a new pattern of movement, such as in the present mirror MA task. While 
the PP group used the kinetic feedbacks delivered during practice, as well as an effector-dependent representation, the participants engaged in MI improved their motor performance without being directly confronted with the task constraints elicited by the mirror condition.

The present data did not reveal any difference in performance gains between the EyesMI and NoEyesMI groups. This finding slightly differs from those reported by Heremans, Helsen, and Feys (2008) and Heremans, Helsen, de Poel, Alaerts, Meyns, and Feys (2009), who proposed that eye movements assist in the process of mental image generation, and therefore reflect the spatiotemporal evolution of the underlying mental processes (Laeng \& Teodorescu, 2002; Mast \& Kosslyn, 2002). Accordingly, these authors demonstrated that the coupling between neural patterns for eye and hand movements remained intact when hand movements were merely imagined as opposed to being physically executed. Eye movements during MI with eyes open or closed showed remarkable similarities to those recorded during PP. Differences between the task designs may be the main explanation, as they investigated aiming wrist flexion-extension movements between targets that were ordered by a metronome. By contrast, Gueugneau, Crognier and Papaxanthis (2008) reported that the accuracy of motor representations was not dependent on the presence or lack of eye movements. In accordance with these latter findings, our results show that imagined performance was not related to eye movements, both in the normal and mirror conditions. It might suggest that the functional role of eye movements during MI did not serve as an objective and real-time indicator of what the participants were actually doing during imagery, nor of what they planned to do to overcome the environmental constraints during the mirror condition. The lack of a role of eye movements in the present experiment could also be explained by some methodological arguments. Firstly, we did not found any group effect when comparing movement accuracy, hence suggesting that the task was maybe too easy, that the limited number of participants and amount of training could have been insufficient, or that the parameter for accuracy was not very sensitive. From this viewpoint, the fact that no effect of eye movements was found on the temporal aspects of a task, does not necessarily imply that eye movements have no role during imagery on other aspects such as accuracy. Another reason why no effect of eye movements was found might be the choice of the task. Although the mirror-reversed task is useful to study new or corrected configurations of actions, it is also an unnatural task, requiring an unnatural uncoupling of eye and hand movements since the cursor (providing visual information) and the hand (providing kinesthetic information) move in opposite directions. This might suggest that there can be a difference between the role of eye movements for imagery of mirror and non-mirror reversed tasks. Finally, since the participants of the NoeyesMI group were asked to fixate at the center of the screen, it cannot be completely excluded that peripheral vision could have been used. Overall, future experimental research remains necessary to clarify the specific role of eye movements during MI, and most especially during MA tasks including mirror reversal movements. The distinction that can be seen in Fig. 2 between the movement times of the normal and mirror conditions in both the PP and EyesMI group, but not in the NoEyesMI group, might further suggest that eye movements could play a role during MI, despite the lack of difference between the two imagery groups in the present experiment.

From a practical viewpoint, the results of the present study may have some implications during motor (re) learning and motor recovery (de Vries \& Mulder, 2007; Munzert, Lorey, \& Zentgraf, 2009). As mirror reversal can be learned through MI, this latter technique could be particularly useful to (re)acquire patterns of movement including new or corrected configuration of actions. In the case of motor impairments, the movement is sometimes impossible, and engaging in MI practice without being directly confronted with the physical constraints of the task might be of strong interest. Although it is premature to draw final conclusion, and though we did not investigate the effects of imagery in patients, the present findings suggest that mirror imagery might be a valuable technique to acquire new motor coordination patterns. Such hypothesis is waiting for experimental investigation, but has received support from a recent study where a patient with spinal cord injury benefited from MI practice after a surgical procedure alleviating the paralysis of the triceps by transferring the distal insertion of either the deltoid or biceps brachii onto the triceps tendon (Grangeon, Guillot, Sancho, Picot, Revol, Rode, \& Collet, 2010). Determining how MI affects the acquisition of mirror reversal patterns of movements will be an exciting focus of research in the coming years.

\section{Appendix}

The following guidelines were given in the imagery script: "Attempt to imagine yourself performing the pursuit tracking task by visualizing the different movements of the joystick as if you had a camera on your head. You see and feel only what you would do if you actually executed the sequence. Pay attention to each joystick movement and make sure to track the moving visual target within the spatial delimited pathway as fast and accurately as possible. Just feel yourself going through the different steps of the task, keeping in mind the correct direction of the joystick". 


\section{References}

Agostino, R., Sanes, J. N., \& Hallett, M. (1996). Motor skill learning in Parkinson's disease. Journal of the Neurological Sciences, 139, $218-226$.

Aizenstein, H. J., Stenger, V. A., Cochran, J., Clark, K., Johnson, M., Nebes, R. D., et al. (2004). Regional brain activation during concurrent implicit and explicit sequence learning. Cerebral Cortex, 14, 199-208.

Atkinson, A. C. (1985). Plots, transformations, and regression. Oxford University Press.

Bock, O., Schneider, S., \& Bloomberg, J. (2001). Conditions for interference versus facilitation during sequential sensorimotor adaptation. Experimental Brain Research, 138, 359-365.

Bootzin, R. R., Manber, R., Loewy, D. H., Kuo, T. F., \& Franzen, P. L. (2004). Sleep disorders. In P. B. Sutker \& H. E. Adams (Eds.), Comprehensive handbook of psychopathology, Chapter 24 (3rd ed., pp. 671-713). New York: Springer.

Brashers-Krug, T., Shadmehr, R., \& Bizzi, E. (1996). Consolidation in human motor memory. Nature, 382, 252-255.

de Vries, S., \& Mulder, T. (2007). Motor imagery and stroke rehabilitation: A critical discussion. Journal of Rehabilitation Medicine, 39, 5-13.

Debarnot, U., Creveaux, T., Collet, C., Doyon, J., \& Guillot, A. (2009). Contribution of sleep to motor consolidation: A motor imagery study. Sleep, 32, 1559-1565.

Debarnot, U., Creveaux, T., Collet, C., Gemignani, A., Massarelli, R., Doyon, J., et al. (2009). Sleep-related improvements in motor learning following mental practice. Brain and Cognition, 69, 398-405.

Decety, J., \& Jeannerod, M. (1996). Mentally simulated movements in virtual reality: Does Fitts's law hold in motor imagery? Behavioural Brain Research, 72, 127-134.

Doyon, J., \& Benali, H. (2005). Reorganization and plasticity in the adult brain during learning of motor skills. Current Opinion in Neurobiology, 15, 161-167.

Doyon, J., Korman, M., Morin, A., Dostie, V., Hadj Tahar, A., Benali, H., et al. (2009). Contribution of night and day sleep vs. simple passage of time to the consolidation of motor sequence and visuomotor adaptation learning. Experiment Brain Research, $195,15-26$.

Doyon, J., Penhune, V., \& Ungerleider, L. G. (2003). Distinct contribution of the cortico-striatal and cortico-cerebellar systems to motor skill learning. Neuropsychologia, 41, 252-262.

Doyon, J., \& Ungerleider, L. G. (2002). Functional anatomy of motor skill learning. In L. R. Squire \& D. L. Schacter (Eds.), Neuropsychology of memory (3rd ed., pp. 225-238). New York: Guilford.

Farah, M. J. (1984). The neurological basis of mental imagery: A componential analysis. Cognition, 18, 245-272.

Fox, J. (2005). The R commander: A basic-statistics graphical user interface to R. Journal of Statistical Software, 19, 1-42.

Gentili, R., Papaxanthis, C., \& Pozzo, D. T. (2006). Improvement and generalization of arm motor performance through motor imagery practice. Neuroscience, 137, 761-772.

Gerardin, E., Sirigu, A., Lehéricy, S., Poline, J. B., Gaymard, B., Marsault, C., et al. (2000). Partially overlapping neural networks for real and imagined hand movements. Cerebral Cortex, 10, $1093-1104$.

Ghilardi, M. F., Eidelberg, D., Silvestri, G., \& Ghez, C. (2003). The differential effect of PD and normal aging on early explicit sequence learning. Neurology, 60, 1313-1319.

Goedert, K. M., \& Willingham, D. B. (2002). Patterns of interference in sequence learning and prism adaptation inconsistent with the consolidation hypothesis. Learning \& Memory, 9, 279-292.
Grangeon, M., Guillot, A., Sancho, P. O., Picot, M., Revol, P., Rode, G., \& Collet, C. (2010). Rehabilitation of the elbow extension with motor imagery in a patient with tetraplegia after a tendon transfer. Arch Phys Med Rehab (in revision).

Gueugneau, N., Crognier, L., \& Papaxanthis, C. (2008). The influence of eye movements on the temporal features of executed and imagined arm movements. Brain Research, 1187, 95-102.

Guillot, A., \& Collet, C. (2005). Duration of mentally simulated movement: A review. Journal of Motor Behaviour, 37, 10-20.

Guillot, A., \& Collet, C. (2008). Construction of the motor imagery integrative model in sport: A review and theoretical investigation of motor imagery use. International Review of Sport and Exercise Psychology, 1, 32-44.

Guillot, A., Collet, C., Nguyen, V. A., Malouin, F., Richards, C., \& Doyon, J. (2008). Functional neuroanatomical networks associated with expertise in motor imagery ability. Neuroimage, 41, 1471-1483.

Guillot, A., Louis, M., \& Collet, C. (2010). Neurophysiological substrates of motor imagery ability. In A. Guillot and C. Collet (Eds). The neurophysiological foundations of mental and motor imagery, (pp. 109-124) Oxford University Press.

Hall, C., \& Martin, K. (1997). Measuring movement imagery abilities: A revision of the movement imagery questionnaire. Journal of Mental Imagery, 21, 143-154.

Helene, A. F., \& Xavier, G. F. (2006). Working memory and acquisition of implicit knowledge by imagery training, without actual task performance. Neuroscience, 139, 401-413.

Heremans, E., Helsen, W. F., de Poel, W. F., Alaerts, K., Meyns, P., \& Feys, P. (2009). Facilitation of motor imagery through movement-related cueing. Brain Research, 1278, 50-56.

Heremans, E., Helsen, W. H., \& Feys, P. (2008). The eye as a mirror of our thoughts: Quantification of motor imagery of goal-directed movements through eye movement registration. Behavioural Brain Research, 187, 351-360.

Hikosaka, O., Nakamura, K., Sakai, K., \& Nakahara, H. (2002). Central mechanisms of motor skill learning. Current Opinion in Neurobiology, 12, 217-222.

Hoddes, E., Dement, W. C., \& Zarcone, V. (1972). The development and use of the Stanford sleepiness scale. Psychophysiology, 9, 150-161.

Holmes, P. S., \& Collins, D. J. (2001). The PETTLEP approach to motor imagery: A functional equivalence model for sport psychologists. Journal of Applied Sport Psychology, 13, 60-83.

Jeannerod, M. (1994). The representing brain: Neural correlates of motor intention and imagery. Behavioural Brain Sciences, 17, $187-245$.

Jeannerod, M. (1995). Mental imagery in the motor context. Neuropsychologia, 33, 1419-1432.

Korman, M., Doyon, J., Doljansky, J., Carrier, J., Dagan, Y., \& Karni, A. (2007). Daytime sleep condenses the time course of motor memory consolidation. Nature Neuroscience, 10, 1206-1213.

Koslow, R. E. (1987). Sex-Related differences and visual-spatial mental imagery as factors affecting symbolic motor skill acquisition. Sex Roles, 17, 521-527.

Krakauer, J. W., Ghilardi, M. F., \& Ghez, C. (1999). Independent learning of internal models for kinematic and dynamic control of reaching. Nature Neuroscience, 2, 1026-1031.

Laeng, B., \& Teodorescu, D. S. (2002). Eye scanpaths during visual imagery reenact those of perception of the same visual scene. Cognitive Science, 26, 207-231.

Laforce, R., \& Doyon, J. (2002). Differential role for the striatum and cerebellum in response to novel movements using a motor learning paradigm. Neuropsychologia, 40, 512-517.

Lotze, M., \& Halsband, U. (2006). Motor imagery. Journal of Physiology - Paris, 99, 386-395.

Malouin, F., Richards, C. L., Jackson, P. L., Lafleur, M. F., Durand, A., \& Doyon, J. (2007). The kinesthetic and visual imagery questionnaire (KVIQ) for assessing motor imagery in persons 
with physical disabilities: A reliability and construct validity study. Journal of Neurologic Physical Therapy, 31, 20-29.

Mast, F. W., \& Kosslyn, S. M. (2002). Eye movements during visual mental imagery. Trends in Cognitive Sciences, 6, 271-272.

Munzert, J., Lorey, B., \& Zentgraf, K. (2009). Cognitive motor processes: The role of motor imagery in the study of motor representations. Brain Research Reviews, 60, 306-326.

Oldfield, R. C. (1971). The assessment and analysis of handedness: The Edinburgh inventory. Neuropsychology, 9, 97-113.

Overduin, S. A., Richardson, A. G., Bizzi, E., \& Press, D. Z. (2008). Simultaneous sensorimotor adaptation and sequence learning. Experimental Brain Research, 184, 451-456.

Papadelis, C., Kourtidou-Papadeli, C., Bamidis, P., \& Albani, M. (2007). Effects of imagery training on cognitive performance and use of physiological measures as an assessment tool of mental effort. Brain and Cognition, 64, 74-85.

Personnier, P., Ballay, Y., \& Papaxanthis, C. (2010). Mentally represented motor actions in normal aging: III. Electromyographic features of imagined arm movements. Behavioural Brain Research, 206, 184-191.

Press, D. Z., Casement, M. D., Pascual-Leone, A., \& Robertson, E. M. (2005). The time course of off-line motor sequence learning. Cognitive Brain Research, 25, 375-378.

Richardson, A. G., Overduin, S. A., Valero-Cabre, A., PadoaSchioppa, C., Pascual-Leone, A., Bizzi, E., et al. (2006). Disruption of primary motor cortex before learning impairs memory of movement dynamics. The Journal of Neuroscience, 26, 12466-12470.

Roberts, R., Callow, N., Hardy, L., Markland, D., \& Bringer, J. (2008). Movement imagery ability: Development and assessment of a revised version of the vividness of movement imagery questionnaire. Journal of Sport \& Exercise Psychology, 30, 200-221.

Seidler, R. D. (2004). Multiple motor learning experiences enhance motor adaptability. Journal of Cognitive Neuroscience, 16, 65-73.
Seidler, R. D. (2006). Differential effects of age on sequence learning and sensorimotor adaptation. Brain Research Bulletin, 70, 337 346.

Sharma, N., Pomeroy, V. M., \& Baron, J. C. (2006). Motor imagery: A backdoor to the motor system after stroke? Stroke, 37, 1941-1952.

Slifkin, A. B. (2008). High loads induce differences between actual and imagined movement duration. Experimental Brain Research, 185, 297-307.

Solodkin, A., Hlustik, P., Chen, E. E., \& Small, S. L. (2004). Fine modulation in network activation during motor execution and motor imagery. Cerebral Cortex, 14, 1246-1255.

Stephan, K. M., Fink, G. R., Passingham, R. E., Silbersweig, D., Ceballos-Baumann, A. O., Frith, C. D., et al. (1995). Functional anatomy of the mental representation of upper extremity movements in healthy subjects. Journal of Neurophysiology, 73, 373-386.

Stickgold, R., \& Walker, M. P. (2007). Sleep-dependent memory consolidation and reconsolidation. Sleep Medicine, 8, 331-343.

Walker, M. P., Brakefield, T., Hobson, J. A., \& Stickgold, R. (2003). Dissociable stages of human memory consolidation and reconsolidation. Nature, 425, 616-620.

Willingham, D. B. (1998). A neuropsychological theory of motor skill learning. Psychological Review, 105, 558-584.

Wohldmann, E. L., Healy, A. F., \& Bourne, L. E. (2007). Pushing the limits of imagination: Mental practice for learning sequences. Journal of Experimental Psychology. Learning, Memory, and Cognition, 33, 254-261.

Wohldmann, E. L., Healy, A. F., \& Bourne, L. E. (2008). A mental practice superiority effect: Less retroactive interference and more transfer than physical practice. Journal of Experimental Psychology. Learning, Memory, and Cognition, 34, 823-833.

Yágüez, L., Nagel, D., Hoffman, H., Canavan, A. G., Wist, E., \& Hömberg, V. (1998). A mental route to motor learning: Improving trajectorial kinematics through imagery training. Behavioural Brain Research, 90, 95-106. 